\title{
Optimized hourglass-shaped resonators for efficient thermal tuning of CROW filters with reduced crosstalk
}

\author{
J. Müller, ${ }^{1}$ A. Zazzi, ${ }^{1}$ G. Vasudevan Rajeswari, ${ }^{1}$ A. Moscoso Mártir, ${ }^{1}$ A. Tabatabaei Mashayekh, ${ }^{1}$ A. D. \\ Das, ${ }^{1}$ F. Merget, ${ }^{1}$ and J. Witzens ${ }^{1}$ \\ ${ }^{1}$ Institute of Integrated Photonics, RWTH Aachen University, Campus Boulevard 73, 52074 Aachen, Germany
}

We present optimized hourglass-shaped resonator CROW filters showing an improved power consumption of up to $30 \%$ when compared with standard circular ring shapes. Metal and doped waveguide heaters are compared in terms of efficiency, crosstalk and insertion losses.

\section{INTRODUCTION}

The high frequency selectivity of ring resonators usually makes it necessary to thermally tune silicon photonics ringbased devices to compensate for thermal fluctuations, given the high thermo-optic coefficient of silicon, and mismatches from the device design due to fabrication tolerances. Thermal tuners typically make up for a large portion of the power consumption, so that optimizing them is paramount to not unnecessarily burden the overall system power consumption. Thermal tuning efficiencies can be improved by removing the substrate below the device or etching trenches on its side in order to increase the thermal isolation [1], however, these process steps are not always available in commercial SOI platforms. Therefore, efficient resonator layout and heater design are crucial to minimize power consumption. In many applications, e.g. optical communications [2],[3], high-speed signal processing [4] and optical switching [5], higher-order filters have been used to implement transfer functions with steeper roll-offs (Chebyshev) or flattop passbands (Butterworth). Coupled resonator optical waveguides (CROW) are commonly used [6]. For these, it is crucial that the resonance frequencies of individual rings are precisely aligned. If the rings of a CROW filter are strongly coupled, as required for large passbands exceeding the fabrication induced spread in resonance frequencies, the rings constituting a CROW do not need to be individually tuned [7]. However, individual tuning of each resonator is necessary for narrower transfer functions. Thermal crosstalk resulting from the close proximity of the rings should then be minimized to increase the efficiency and decrease the complexity of thermal control [8].

\section{Devices AND Thermal Tuner Design}

Here, our objective is to realize a bank of $3^{\text {rd }}$ order CROW filters dividing a wideband signal into eight individual slices of $30 \mathrm{GHz}$ each, as part of the optical processing in an integrated photonically-assisted broadband ADC [4],[9]. The free spectral range (FSR) needs to be of at least $240 \mathrm{GHz}(8 \times 30 \mathrm{GHz})$. A larger than required FSR corresponding in smaller than required resonator circumference reduces the footprint, however we chose to keep it close to its required minimum as a bigger circumference facilitates the customization of the resonator shape, in particular in view of otherwise occurring bending losses. Due to the relatively low $30 \mathrm{GHz}$ passband, the insertion losses (IL) of the filter are quite sensitive on waveguide and excess coupler losses and tuning of individual rings is required. Since no dedicated heater layer was available in the selected platform, we have pursued two alternative approaches. In the first implementation, we opted for n-doping the waveguides along $16 \%$ of the $305.9 \mu \mathrm{m}$ long resonator circumference, realizing a resistive element directly heating the $400 \mathrm{~nm}$ wide and $220 \mathrm{~nm}$ thick waveguide, see Fig. 1 (a). Electrical contacting is enabled by a rib waveguide geometry with a $100 \mathrm{~nm}$ thick slab. The resistor is formed by a reduced concentration n-doped region with an electrical material conductivity of $4.2 \times 10^{3} \mathrm{~S} / \mathrm{m}$ applied to the waveguide and to the slab over a total width of $1.6 \mu \mathrm{m}$, beyond which the doping concentration is increased for electrical transport and contacting. Thermal simulations show that the length of the doped section does not substantially impact the thermal phase tuner's efficiency. Therefore, we have only doped a small section to keep additional waveguide losses low, constrained on the low end from further shrinking by maintaining a small enough current density to not damage the device by electromigration when tuning by a full FSR. In the second implementation, we have used the metal layer closest to the waveguide, consisting of aluminum, to form a resistive element above the waveguide. In order to ensure the voltage to be dropped across the heater section, rather than across the interconnects, we have used a meandered structure with a stripe width of $360 \mathrm{~nm}$, as shown in Fig. 1 (b). Here, we have been able to implement a fully etched waveguide since no electrical contacting of the silicon layer is necessary. For both implementations, we have divided the ring into a tuning and a coupling section. In the tuning section, we have brought opposite waveguides together as close as possible without optically coupling them, leaving a gap of $3 \mu \mathrm{m}$ for the ring structure with metal heater and $7 \mu \mathrm{m}$ for the structure with doped waveguide heater (constrained by the ground contacts having to be placed between the waveguides). Due to the large $16 \mu \mathrm{m}$ bends needed for the rib waveguides to not incur excessive bending losses, the waveguides in the heater section run in parallel for $11.5 \mu \mathrm{m}$ only (doped heaters), whereas they cover a length of $60 \mu \mathrm{m}$ in the stronger confined fully etched ridge waveguide (metal heaters) with a bending radius of only $5 \mu \mathrm{m}$. The 
heaters have not been extended to the coupling sections to minimize thermal crosstalk and keep the coupling strengths constant, maintaining a distance of $36 \mu \mathrm{m}$ to the next ring for the doped waveguide heaters. For the structure with metal heaters, this distance was reduced to $9.7 \mu \mathrm{m}$ as a consequence of the tighter bends.

\section{EXPERIMENTAL RESULTS AND FURTHER OPTIMIZATION}

The doped waveguide (metal) tuner features a resistance of $82 \Omega(171 \Omega$ ) and a tuning efficiency measured to be 50 $\mathrm{mW} / \mathrm{FSR}$ (47 mW/FSR) for a single ring, revealing almost equal efficiency for both. Thermal simulations (Fig. 1(c)) indicate that tuning rings with an hourglass shape requires about $30 \%$ less power than tuning rings with a conventional circular shape. Simulated thermal crosstalk was as low as $1.8 \%$ for the doped waveguide heater and $7.3 \%$ for the metal heater. Adopting the doped heater, waveguide losses averaged over the entire circumference increase by 4 $\mathrm{dB} / \mathrm{cm}$, leading to $3 \mathrm{~dB}$ IL for the entire 3-ring CROW filter, as opposed to $1.5 \mathrm{~dB}$ for the metal heater solution.

Simulations indicate further potential for optimization of the heater performance. As mentioned above, the length of the doped waveguide does not significantly impact the tuner efficiency, whereas the slab height plays a major role due to its impact on the thermal resistance experienced by the waveguide. A slab of only $50 \mathrm{~nm}$ would reduce the power per FSR by $7.5 \%$ and the crosstalk to $1.6 \%$. In case of the metal heater, obviously, significant improvement can be obtained by reducing the distance between heater and waveguide. According to simulations, a distance between heater and waveguide of $600 \mathrm{~nm}$ would result in only $0.2 \mathrm{~dB} / \mathrm{cm}$ additional waveguide losses while reducing the tuning power per FSR by about $16 \%$ and the crosstalk to $5.2 \%$. Figure 1(d) shows the CROW transfer function before and after alignment, with the desired transfer function obtained in the final alignment (green) also corresponding to the maximization of the dropped power from a pilot tone centered in the desired filter passband [10]. To prevent the final filter shape from drifting, alignment is maintained by an autonomous control system.

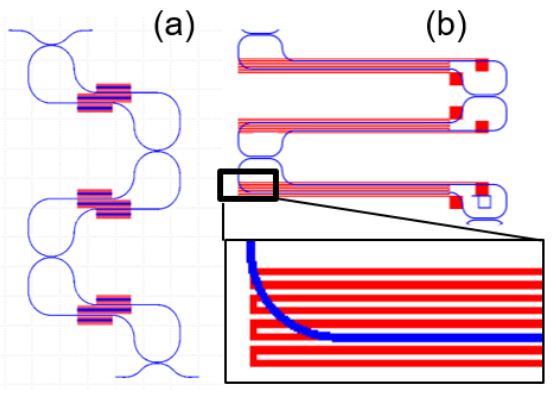

(c)

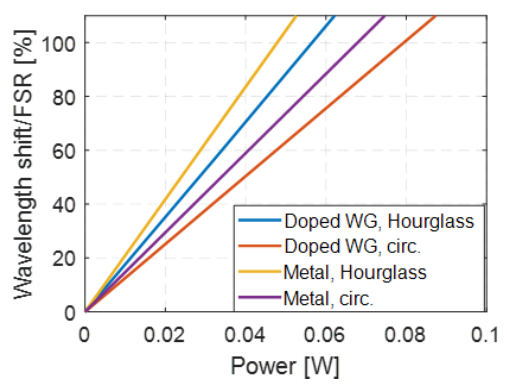

(d)

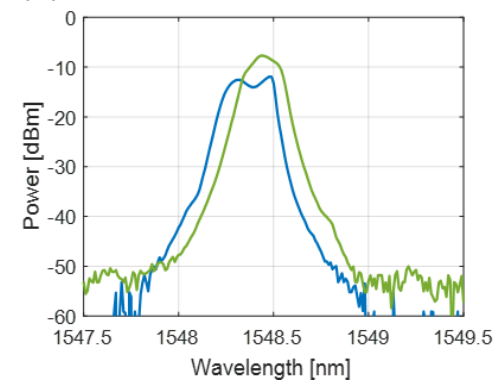

Fig. 1. CROW filters divided into a coupling and a tuning section, implemented with (a) a doped waveguide heater and (b) a meandered aluminum heater on top of the waveguide. (c) Simulated tuning efficiencies compared for the optimized hourglass and the conventional circular ring shape for both heater implementations. (d) CROW filter transfer function before (blue) and after (green) alignment.

\section{CONCLUSIONS}

We have shown that an optimized hourglass resonator shape reduces the tuning power per FSR by about $30 \%$ when compared to a conventional circular shape. The best choice of thermal phase tuner depends on fabrication constraints as well as requirements on acceptable insertion losses, tuning efficiency and crosstalk. Metal heaters result in very low excess waveguide loss and can be fabricated very reliably without suffering from doping concentration variations. Due to the high flexibility in routing waveguides and since no slab is needed, metal heaters are potentially more efficient given the availability of a metal layer close enough to the silicon device layer. In terms of crosstalk, direct heating of the waveguides showed better results as a consequence of the larger distance between phase shifters, as implemented in the layouts, as well as the phase shifters themselves being more compact.

[1] P. Dong et al., "Thermally tunable silicon racetrack resonators with ultralow tuning power," Opt. Expr., vol. 18, pp. 20298-20304, 2010.

[2] J. Koch et al., "Experimental Demonstration of a Silicon-Photonics WDM NLFT Soliton Transmitter," in Proc. OFC., Art. ID W6A.34, 2021.

[3] A. Moscoso-Mártir et al., "8-channel WDM silicon photonics transceiver with SOA and semiconductor mode-locked laser," Opt. Expr., vol. 26, pp. 25446-25459, 2017.

[4] D. Fang et al., "Optical Arbitrary Waveform Measurement (OAWM) on the Silicon Photonic Platform," in Proc. OFC, Art. ID F2E.6, 2021.

[5] S. Cho et al., "Apodized SCISSORs for filtering and switching," Opt. Expr., vol. 16, pp. 19078-19090, 2008.

[6] A. Melloni et al., "Synthesis of direct-coupled-resonators bandpass filters for WDM systems," J. Lightwave Technol. vol. 20, pp. 296-303, 2002.

[7] S. Romero Garcia et al., "Wideband multi-stage CROW filers with relaxed fabrication tolerances," Opt. Expr., vol. 26, pp. 4723-4737, 2018.

[8] M. Milanizadeh et al., "Canceling Thermal Cross-Talk Effects in Photonic Integrated Circuits," J. Lightwave Technol., vol. 37, pp. 1325$1332,2019$.

[9] A. Zazzi et al., "Fundamental limitations of spectrally-sliced optically enabled data converters arising from MLL timing jitter," Opt. Expr., vol. 28, pp. 18790-18813, 2020.

[10] J. C. C. Mak et al., "Automatic Resonance Alignment of High-Order Microring Filters," J. Quant. Electron., vol. 15, no. 11, Art. ID 0600411, 2015. 